\title{
Efetividade da estimulação elétrica funcional no membro superior de hemiparéticos crônicos
}

\author{
Effectiveness of functional electrical stimulation in chronic hemiparetic upper limb \\ Felipe Brandão de Rezende' ${ }^{1}$, Heloise Cazangi Borges², Vanessa \\ Costa Monteiro ${ }^{3}$, Danilo Masiero ${ }^{4}$, Therezinha Rosane Chamlian ${ }^{5}$
}

\begin{abstract}
RESUMO
Objetivos. Verificar a efetividade da EEF aplicada em extensores de cotovelo e punho do membro superior de hemiparéticos crônicos para melhora da força, ADM (arco dinâmico de movimento), espasticidade e funcionalidade do membro. Método. Trata-se de um relato de casos em que três indivíduos (dois do sexo feminino e um do sexo masculino), apresentando hemiparesia crônica com predomínio braquiofacial e faixa etária entre 56 e 64 anos foram submetidos à aplicação de EEF com estimulação simultânea de extensores de punho e cotovelo por 30 minutos diários, duas vezes por semana, durante seis semanas. Foi feita avaliação da força, $A D M$, espasticidade e funcionalidade de membro hemiparético antes e após intervenção e também no segmento de cinco semanas após final da terapia. Resultados. Todos os pacientes apresentaram melhora na força, ADM e funcionalidade, e destes, dois apresentaram redução de espasticidade. $\mathrm{Na}$ avaliação de cinco semanas, verificou-se que apenas o quesito força não se manteve. Conclusão. A aplicação deste novo parâmetro de EEF com estimulação simultânea de extensores de punho e cotovelo mostrou-se benéfica para melhora funcional do membro superior de pacientes hemiparéticos crônicos.
\end{abstract}

Unitermos. Estimulação Elétrica, Hemiparesia, Acidente Cerebrovascular, Membro Superior.

Citação. Rezende FB, Borges HC, Monteiro VC, Masiero D, Chamlian TR. Efetividade da estimulação elétrica funcional no membro superior de hemiparéticos crônicos.

Trabalho realizado no Centro de Reabilitação Lar Escola São Francisco/ UNIFESP-EPM, São Paulo-SP, Brasil.

1. 1.Fisioterapeuta especializando em Fisioterapia Motora Hospitalar e Ambulatorial aplicada à Neurologia da Universidade Federal de São Paulo - Unifesp. São Paulo-SP, Brasil.

2. 2.Fisioterapeuta do Lar Escola São Francisco - LESF, coordenadora e preceptora do curso de Especialização Fisioterapia Motora Hospitalar e Ambulatorial aplicada à Neurologia da Unifesp. São Paulo-SP, Brasil.

3. 3.Fisioterapeuta do LESF, preceptora do curso de Especialização Fisioterapia Motora Hospitalar e Ambulatorial aplicada à Neurologia da Unifesp. São Paulo-SP, Brasil.

4. 4.Ortopedista, Doutor, Professor Associado e Vice Chefe da Disciplina de Fisiatria do Departamento de Ortopedia e Traumatologia da Unifesp. São Paulo-SP, Brasil.

5. 5.Fisiatra, Doutora, Professora Afiliada e Chefe da Disciplina de Fisiatria do Departamento de Ortopedia e Traumatologia da Unifesp. São Paulo-SP, Brasil.

\section{SUMMARY}

Objective. To verify the effectiveness of the FES applied in wrist and elbow extensors in chronic hemiparetic upper-extremity to improve power, spasticity, dynamic range of motion (ROM) and functionality. Method. This is a cases report, in that three subjects (two female and one male), presenting chronic upper limb hemiparesis and age range of 56 to 64 years were submitted a treatment with FES in wrist and elbow extensors simultaneously. This was applied during 30 minutes per day, two days of week, in a total of six weeks. As evaluation the measurement of upper-extremity power, ROM, spasticity and functionality was made before and after the treatment. A five weeks follow-up was carried too. Results. All the patients showed improvement in the upper limb power, ROM and functionality. Two subjects showed reduction in spasticity. Just the force wasn't remained throughout follow-up period. Conclusion. The application of FES with simultaneous stimulation of wrist and elbow extensors revealed beneficial for functional improvement of the chronic hemiparetic upper limb.

Keywords. Electric Stimulation, Hemiparesis, Stroke, Upper Extremity.

Citação. Rezende FB, Borges HC, Monteiro VC, Masiero D, Chamlian TR. Effectiveness of functional electrical stimulation in chronic hemiparetic upper limb.
Endereço para correspondência: Felipe B Rezende

R. Santa Cruz, 1021/73C CEP 04121-001, São Paulo-SP, Brasil e-mail: felipe_br28@yahoo.com.br

Relato de Caso Recebido em: 04/10/2007 Revisão: 05/10/2007 a 03/03/2008

Aceito em: 04/03/2008 Conflito de interesses: não 


\section{INTRODUÇÃO}

O Acidente Vascular Encefálico (AVE) pode ser designado como sendo um tipo de doença cerebrovascular caracterizada pela lesão encefálica decorrente do comprometimento permanente no fluxo sanguíneo local, acarretando em déficits sensitivos e motores que perduram por mais de 24 horas ${ }^{1}$. As doenças cérebrovasculares representam a primeira causa de morte no Brasil, sendo 1/3 dos óbitos atribuídos às mesmas no período entre 1980 a $1995^{2}$.

É relatado que, mesmo após o período de recuperação espontânea durante os primeiros 6 meses, mais de $85 \%$ dos indivíduos que sofreram AVE apresentam déficits residuais no membro superior, em graus variados, sendo que na maioria dos casos a recuperação funcional ocorre de forma insatisfatória ${ }^{3}$.

Em relação aos déficits do membro superior parético, uma pesquisa realizada por Golebatch e Gandevia observou que os grupos musculares distais encontram-se mais afetados que os músculos proximais, sendo os extensores do punho e dedos os grupos musculares mais fracos ${ }^{4}$. No que diz respeito aos grupos musculares proximais, verificou-se que os extensores do cotovelo são os que apresentam menor torque. Segundo Muellbacher e colaboradores existe uma competitividade por áreas corticais entre as partes do membro superior afetado $^{5}$. A preservação parcial de vias responsáveis pela motricidade do segmento proximal do membro superior possibilita uma expansão da representatividade cortical correspondente à motricidade proximal, com conseqüente diminuição da representatividade do segmento distal.

Vários estudos que evidenciam métodos terapêuticos que objetivam a recuperação motora do membro superior comprometido têm mostrado que a eletro-estimulação muscular pode ser útil para melhora da força, arco dinâmico de movimento (ADM), funcionalidade e redução do tônus espástico em pacientes hemiparéticos $\operatorname{crônicos}^{5-12}$.

Uma das principais modalidades de eletro-estimulação é a estimulação elétrica funcional (EEF), que consiste na estimulação transcutânea do músculo privado de controle normal, associado a realização de atividades funcionais ${ }^{12}$.

Algumas pesquisas propõem que, os estímulos sensoriais que chegam ao córtex provindos da ativação muscular do membro afetado, exercem influência direta na ativação motora e que a realização associada de atividades funcionais (atividades motoras que exijam o uso de habilidade) potencializa a reorganização cortical ${ }^{13}$. Contudo, todos os trabalhos objetivaram relatar somente os resultados das aplicações da eletro-estimulação em extensores de punho e dedos.

Com relação aos mecanismos neuromusculares de motricidade do membro superior, é descrito que as articulações do cotovelo e punho são controladas como uma unidade sinérgica e que os movimentos conjugados de flexão e extensão do cotovelo e punho permitem melhor ativação dos grupos musculares agonistas do movimento ${ }^{14}$.

A análise dos mecanismos neuromusculares de motricidade do membro superior, em associação a técnicas que permitem maximizar a função e motricidade do membro superior deficitário, sugerem novos meios de abordagem terapêuticas para obtenção de melhores resultados.

Portanto, este trabalho tem por objetivo verificar a efetividade da EEF aplicada em extensores de cotovelo e punho, associada à realização de atividades funcionais, no membro superior de hemiparéticos crônicos para melhora da força muscular, $A D M$, espasticidade e funcionalidade do membro.

\section{MÉTODO}

Trata-se de um trabalho prospectivo experimental unicêntrico, em forma de relato de casos, realizado no Setor de Fisioterapia da disciplina de fisiatria do Departamento de Ortopedia e Traumatologia da Unifesp e no LESF. O estudo foi aprovado pelo Comitê de Ética em Pesquisa da Unifesp (processo 2006060882017).

\section{Amostra}

Participaram deste trabalho indivíduos portadores de AVE que se encontravam em tratamento multidisciplinar (fisioterapia, fonoaudiologia e fisiatria) e/ou pacientes que já foram atendidos na Instituição.

Foram considerados critérios de inclusão: ser portador de hemiparesia decorrente de AVE isquêmico; apresentar hemiparesia desproporcionada com predomínio braquial; 1 ano ou mais de tempo decorrido após evento agudo a doença (AVE crônico), visto que o platô de recuperação espontânea é de 6 meses pós evento agudo; faixa etária entre 40 e 70 anos, visando manter uma amostra mais homogênea e eliminar as possíveis variáveis decorrentes da discrepância de idades; apresentar no mínimo grau 1 de força de extensores de cotovelo 
e punho segundo escala de 5 pontos de Kendall15; e não ter comprometimento osteomuscular prévio que interfira na ADM de extensão de cotovelo, punho e dedos.

Foram considerados critérios de exclusão: apresentar afasia de compreensão e outros comprometimentos que interfiram na compreensão da intervenção; instabilidade clínica (hipertensão arterial e diabetes melitus não controlados); mais de um episódio de AVE; espasticidade superior ao grau 3 pela escala de tônus de Ashworth ${ }^{16}$; estar em tratamento por qualquer outro tipo de terapia física voltada para o membro superior; apresentar outras doenças neurológicas associadas; e possuir tempo de instalação do AVE superior a 6 anos.

\section{Procedimentos}

Foram selecionados 4 participantes através a análise de prontuário e avaliação individual, sendo dois do sexo masculino e dois do sexo feminino. Destes, três possuíam hemiparesia à esquerda e um a direita e, aos mesmos, foi apresentado o termo de consentimento livre e esclarecido. A faixa etária variava entre 56 e 64 anos, tempo pósAVE entre 1 a 6 anos e três destes encontravam-se em tratamento fonoaudiológico e em etapa final de tratamento fisioterapêutico no LESF. Para evitar interferência nos resultados do presente trabalho, foi solicitado aos fisioterapeutas que não realizassem nenhum tipo de abordagem no membro superior dos pacientes selecionados. Todos os pacientes relataram nunca terem sido submetidos a tratamento com EEF.

Durante o período de intervenção, um indivíduo foi excluído do trabalho devido a reação hiperêmica local, permanecendo três pacientes (duas mulheres e um homem) até o final do trabalho.

A intervenção proposta consistiu de aplicação da EEF, com eletro-estimulação em extensores de cotovelo e punho, associado à realização de tarefas específicas. Para tal, foi utilizado o aparelho FESMED $^{\circledR}$ (com duas entradas para disparador manual e duas saídas para eletrodos), na modalidade FES.

Baseando-se nos mecanismos de neuromotricidade do membro superior, foram propostos exercícios visando o treino do agrupamento muscular extensor do membro hemiparético em associação ao uso do membro sadio e de forma isolada ${ }^{17}$. Na primeira atividade o paciente ficava sentado em frente a uma mesa, encaixava cones usando o membro sadio para fixar um cone e o membro hemiparético para conduzir e encaixar o outro cone sobre o fixo. Na segunda tarefa o paciente encontrava-se sentado em frente a uma mesa, segurava um copo de plástico rígido com o membro hemiparético, levava-o em direção a boca e depois o colocava novamente à mesa. E na terceira atividade o paciente ficava sentado em frente a uma mesa, estendia o membro hemiparético para pegar bolas de tênis sobre a mesa e as conduzia para dentro de uma caixa de sapatos ao lado.

Em média, os exercícios foram realizados em uma série de 10 repetições, três vezes cada, totalizando 30 repetições de cada exercício. Entre um exercício e outro, foi concedido descanso de $1 \mathrm{mi}$ nuto ao paciente, ou também entre as repetições dos exercícios em caso de solicitação do paciente. Os pacientes que inicialmente não conseguiam guiar o membro deficitário adequadamente para realização das tarefas recebiam assistência do terapeuta.

Conforme as descrições na literatura de que não existe comprovação de uma relação direta entre o tempo de eletro-estimulação e melhores ganhos de motricidade e que, a realidade brasileira em atendimento de fisioterapia não permite sessões de terapias demasiadamente prolongadas, no presente trabalho foram realizadas sessões de 30 minutos, duas vezes por semana, durante seis semanas $^{18}$. O aparelho foi ajustado no modo manual e a eletro-estimulação foi ativada por meio de disparadores manuais durante a solicitação dos músculos extensores para realização das atividades funcionais; nos períodos de atividade dos músculos flexores a eletro-estimulação não foi ativada. Os demais parâmetros do aparelho foram ajustados da seguinte forma: tempo de subida e de descida igual a 1 segundo, freqüência $35 \mathrm{hz}$, amplitude de pulso de 300 e intensidade de acordo com a tolerância de cada indivíduo.

Os eletrodos foram acoplados ao membro com uso de uma fita adesiva e gel condutor, sendo utilizado o método bipolar de estimulação com um eletrodo proximal e outro distal nos respectivos grupos musculares alvos para realização da extensão de cotovelo, punho e dedos ${ }^{19}$.

A avaliação inicial foi realizada cinco dias antes do início da abordagem proposta e a avaliação final cinco dias depois do término da intervenção. Foi realizada também uma reavaliação após 5 se- 
manas do término do período de intervenção, sendo todas estas feitas por um avaliador independente.

Como parâmetros para mensuração dos resultados foram avaliados os seguintes quesitos: força muscular de extensores de cotovelo e punho pela escala de força de Kendall15; ADM das articulações de cotovelo e punho mensuradas com goniômetro manual; grau de espasticidade de flexores de punho e cotovelo com base na escala modificada de Ashworth 16 e funcionalidade do membro superior avaliada usando a escala de Fulg-Meyer

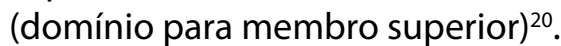

A escala de Kendall consiste de uma avaliação subjetiva da força muscular que utiliza como parâmetros observacionais de desempenho o arco de movimento, realização do movimento com ou sem gravidade ou ainda com aplicação de resistência manual. Apresenta graduação de 5 pontos, em que a pontuação mínima (zero) corresponde a ausência de contração muscular e a pontuação máxima (cinco) corresponde a capacidade que o sujeito tem em realizar o movimento contra a gravidade e com aplicação de resistência manual máxima. Já o domínio motor para membro superior da escala de Fugl-Meyer é um dos cinco domínios desta escala utilizado para mensurar a performance motora, coordenação e ação reflexa do ombro, cotovelo, antebraço, punho e dedos. Cada domínio contém múltiplos sub-tens, sendo estes graduados em forma de uma escala ordinal de 3 pontos $(0=$ não realiza, $1=$ realiza parcialmente, $2=$ realiza totalmente).

\section{RESULTADOS}

\section{Caso 1}

Paciente do sexo feminino, 58 anos, apresentando hemiparesia á direta, leve afasia de expressão e sem comprometimento cognitivo. O laudo médico atestava o diagnóstico de AVE isquêmico de artéria cerebral média esquerda e tempo decorrido 6 anos após evento agudo. Encontrava-se em tratamento fonoaudiológico efisioterapêutico. Não apresentava qualquer outro comprometimento clínico associado. Os resultados das avaliações pré e pós-EEF correspondentes ao caso 1 estão expressos, numericamente, de acordo com o quadro 1.

\section{Caso 2}

Indivíduo de 56 anos, sexo masculino, apresentava hemiparesia à esquerda decorrente de AVE isquêmico de artéria cerebral média, segundo laudo médico. Apresentava um ano de lesão, hipertenso controlado e em tratamento fisioterapêutico no LESF. Os resultados das avaliações pré e pós-EEF correspondentes ao caso 2 estão expressos, numericamente, no quadro 2.

\section{Caso 3}

Paciente de 61 anos, sexo feminino, com hemiparesia à esquerda conseqüente de AVE isquêmico em território da artéria cerebral média direita. Tabagista, apresentava hipertensão arterial controlada e 6 anos de lesão. Já havia sido submetida anteriormente a tratamento fisioterapêutico e de terapia ocupacional no LESF. Os resultados das avaliações pré e pós-EEF correspondentes ao caso 3 estão expressos, numericamente, de acordo com o quadro 3.

Quadro 1. Dados da avaliação do caso 1.

\begin{tabular}{|c|c|c|c|c|c|c|c|}
\hline & \multicolumn{2}{|c|}{ Força } & \multicolumn{2}{|l|}{ ADM } & \multicolumn{2}{|c|}{ Espasticidade } & \multirow[t]{2}{*}{ Fugl-Meyer } \\
\hline & Ex.C & Ex.P & Ex.C & Ex.P & Flx.C & Flx.P & \\
\hline $\mathrm{Ai}$ & 3 & 1 & $10^{\circ} s-f$ & $5^{\circ}$ & 2 & 3 & 10 pontos \\
\hline Af & 3 & 2 & $4^{\circ} s-f$ & $27^{\circ}$ & $1+$ & $1+$ & 18 pontos \\
\hline A5 & 3 & 1 & $0^{\circ}$ & $37^{\circ 1}$ & 1 & 2 & 17 pontos \\
\hline
\end{tabular}

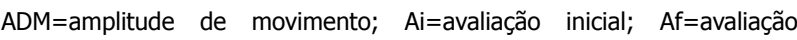
final; $A 5=$ segmento de 5 semanas; Ex.C=extensores de Co-

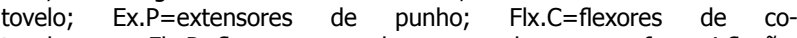
tovelo; Flx.P=flexores de punho; $\mathrm{s}$-f=semi-flexão.

Quadro 2. Dados da avaliação do caso 2.

\begin{tabular}{|c|c|c|c|c|c|c|c|}
\hline & \multicolumn{2}{|c|}{ Força } & \multicolumn{2}{|l|}{ ADM } & \multicolumn{2}{|c|}{ Espasticidade } & \multirow[t]{2}{*}{ Fugl-Meyer } \\
\hline & Ex.C & Ex.P & Ex.C & Ex.P & Flx.C & Flx.P & \\
\hline $\mathrm{Ai}$ & 1 & 1 & $37^{\circ} \mathrm{s}-\mathrm{f}$ & $0^{\circ}$ & $1+$ & 2 & 6 pontos \\
\hline Af & 2 & 1 & $30^{\circ} \mathrm{s}-\mathrm{f}$ & $20^{\circ}$ & $1+$ & 2 & 13 pontos \\
\hline A5 & 1 & 1 & $15^{\circ} s-f$ & $13^{\circ 1}$ & $1+$ & 2 & 13 pontos \\
\hline
\end{tabular}

$\mathrm{ADM}=$ amplitude de movimento; $\mathrm{A}=$ =avaliação inicial; $\mathrm{Af}=$ avaliação final; $A 5=$ segmento de 5 semanas; Ex.C=extensores de cotovelo; Ex.P=extensores de punho; Flx.C=flexores de co-

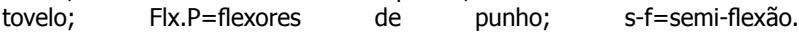

Quadro 3. Dados da avaliação do caso 3.

\begin{tabular}{|c|c|c|c|c|c|c|c|}
\hline & \multicolumn{2}{|c|}{ Força } & \multicolumn{2}{|l|}{ ADM } & \multicolumn{2}{|c|}{ Espasticidade } & \multirow[t]{2}{*}{ Fugl-Meyer } \\
\hline & Ex.C & Ex.P & Ex.C & Ex.P & Flx.C & Flx.P & \\
\hline $\mathrm{Ai}$ & 3 & 4 & $30^{\circ} s-f$ & $20^{\circ}$ & 2 & $1+$ & 31 pontos \\
\hline Af & 3 & 4 & $0^{\circ}$ & $33^{\circ}$ & 1 & $1+$ & 41 pontos \\
\hline A5 & 3 & 4 & $3^{\circ} \mathrm{s}-\mathrm{f}$ & $35^{\circ 1}$ & 1 & $1+$ & 40 pontos \\
\hline
\end{tabular}

ADM=amplitude de movimento; $A \mathrm{i}=$ avaliação inicial; $A \mathrm{f}=$ avaliação final; $A 5=$ segmento de 5 semanas; Ex.C=extensores de cotovelo; Ex.P=extensores de punho; Flx.C=flexores de Cotovelo; $\quad F \mid x . P=f l e x o r e s \quad d e \quad$ punho; $s$-f=semi-flexão. 


\section{DISCUSSÃO}

De acordo com os resultados obtidos, todos os pacientes se beneficiaram da terapia por EEF, apresentando melhora na maioria dos quesitos avaliados quando comparados os valores da avaliação inicial aos valores da avaliação final e do seguimento de 5 semanas.

O primeiro parâmetro avaliado foi a força e, segundo os dados finais, todos obtiveram ganho de força em, pelo menos, um grupo muscular (extensores de punho ou extensores de cotovelo). Entretanto, na avaliação de 5 semanas após o término da terapia, foi verificado que todos os indivíduos voltaram a apresentar valores de força iguais aos da avaliação inicial, indicando que este beneficio obtido não se manteve. Tal desfecho pode ser explicado devido ao pouco uso do membro hemiparético durante as atividades diárias, assim como relatado por Chae et $\mathrm{al}^{8}$, em que foi evidenciado regressão dos ganhos obtidos durante a avaliação do seguimento de três meses pós-tratamento.

Alguns outros trabalhos com EEF mostram uma associação positiva entre esta intervenção e o ganho de força, tal como reportado por Hummelsheim et al, em que foi feita a aplicação de estimulação elétrica neuromuscular associada à contração voluntária de extensores de punho e dedos no membro superior de pacientes hemiparéticos crônicos durante 12 sessões de 30 minutos cada, sendo verificada melhora na força de extensores de punho e de preensão ${ }^{12}$. Da mesma forma, em uma revisão sistemática foi relatado melhora da força muscular de pacientes hemiparéticos crônicos submetidos à EEF e, segundo os autores, a capacidade de produzir força de contração eficaz é uma premissa básica para qualquer movimento. Entretanto, nestes trabalhos não houve avaliação tardia pós-terapia para averiguar se o ganho de força se manteve ${ }^{13}$.

No que diz respeito ao grau de espasticidade, observou-se que o indivíduo 1 apresentou redução do tônus flexor de cotovelo e punho; o indivíduo 3 apresentou redução do tônus flexor de cotovelo, mantendo inalterado o tônus flexor de punho e o indivíduo 2 não apresentou alteração no tônus. Na avaliação do seguimento de cinco semanas foi observado manutenção dos ganhos no caso 3, diminuição em 1 grau no tônus flexor de cotovelo (passou do grau 1+ para grau 1) no caso 2 e aumento em 1 grau no tônus flexor de punho (passou de grau 1+ para grau 2) no caso 3 .
Tais resultados puderam ser melhor evidenciados em uma série de casos desenvolvida por Hummelsheim et al $^{12}$, que verificaram diminuição da espasticidade, e esta, teve relação direta ao expressivo ganho funcional dos indivíduos.

É proposto que a ativação das fibras la, correspondentes ao agrupamento extensor, induz a inibição recíproca dos músculos flexores, provocando seu relaxamento e conseqüente diminuição do tônus ${ }^{16}$. Entretanto, devido à heterogeneidade dos resultados apresentados neste trabalho e ao pequeno número da amostra, não é possível afirmar que o uso da EEF isolada é benéfico para redução do tônus. Alguns fatores não controláveis e peculiares a cada indivíduo, tais como estilo de vida, ambiente de trabalho ou moradia, problemas familiares e estresse mental podem ter sido determinantes para a apresentação heterogênea destes resultados.

Ao ser avaliada a ADM, verificou-se que todos os pacientes apresentaram ganhos marcantes. Os resultados da avaliação no seguimento de 5 semanas indicaram que a maioria das articulações avaliadas mantiveram e, em alguns casos, até aumentaram seus ganhos anteriores. A exceção é feita à ADM para extensão de cotovelo do indivíduo 3 e de punho do indivíduo 2 , que apresentaram uma redução de 3 e 7 graus, respectivamente, em seus ganhos. Contudo, os valores finais para a ADM apresentados pelos pacientes mostraram ser suficientes para realização de atividades funcionais de alcance, com extensão combinada de cotovelo e punho.

Resultados igualmente expressivos foram observados por Baker et al ${ }^{11}$, em que foi evidenciado ganho significativo na ADM em indivíduos hemiparéticos crônicos submetidos a tratamento com estimulação elétrica de extensores de punho e dedos. Ao que tudo indica, a movimentação ativa exercida pela contração muscular estimula a produção de líquido sinovial nas articulações e promove o alongamento capsular, ligamentar e muscular, permitindo ganhos no arco dinâmico de movimento.

A avaliação da funcionalidade do membro foi realizada por meio da utilização da escala de Fulg-Meyer e, de acordo com os valores obtidos, todos os pacientes obtiveram bons índices de meIhora. Os itens em que se podem observar melhoras comuns aos três pacientes correspondem aos seguintes movimentos de acordo com a descrição 
da escala: flexão de ombro e cotovelo e supinação de antebraço, flexão ativa em massa dos dedos e segurar contra resistência. Os pacientes 1 e 2 não apresentaram melhora nos itens relacionados às funções do punho e a coordenação, porém, o paciente 1 demonstrou ganhos um pouco mais expressivos, obtendo melhora nos itens relacionados à abdução, extensão e rotação do membro parético, extensão ativa em massa dos dedos e abdução do polegar e extensão das primeiras articulações metacarpofalangeanas e interfalangeanas. $\mathrm{O}$ indivíduo 3 foi o que obteve mais ganhos, tendo meIhora adicional nos itens relacionados às funções do punho e a coordenação. Na avaliação do seguimento de 5 semanas foi observada uma diminuição irrisória de 1 ponto na escala de Fugl-Meyer para os pacientes 1 e 3 e manutenção da pontuação alcançada pelo paciente 2 .

Resultados semelhantes foram observados em um relato de casos que propunha averiguar a eficácia da aplicação da EENM em extensores de punho e dedos, associada a exercícios ativos de extensão dos mesmos, para melhora funcional do membro superior parético. Neste estudo, os indivíduos com hemiparesia leve e moderada obtiveram melhora na escala de Fugl-Meyer, mas o paciente classificado como hemiparético grave não exibiu melhora nesta escala. Os benefícios obtidos permaneceram constantes na avaliação do seguimento de 12 semanas do mesmo trabalho ${ }^{13}$. De forma semelhante, no presente estudo, o grau de comprometimento motor do membro superior mostrou-se intimamente relacionado à proporção de melhora, pois o indivíduo que apresentou a menor pontuação na escala de Fugl-Meyer durante a avaliação inicial foi o que exibiu menor índice de melhora nesta escala, ocorrendo o oposto com o sujeito que exibiu maior pontuação durante a avaliação inicial.

Em uma revisão, cujo objetivo foi averiguar e quantificar o grau de melhora funcional do membro superior de hemiparéticos submetidos a três modalidades de tratamento (EENM, EEF, facilitação neuromuscular proprioceptiva) ou a nenhum tratamento, foi verificado que a EEF mostrou-se mais eficaz para melhora da funcionalidade, com incremento em $42 \%$ na escala de Fugl-Meyer, contra $25 \%$ e $18 \%$ relacionados, respectivamente, ao uso de EENM e facilitação neuromuscular propriocepi$\mathrm{va}^{14}$. Tal resultado vai ao encontro dos resultados obtidos no presente trabalho, em que se pode ob- servar porcentagem de incremento na escala de Fugl-Meyer semelhante ao trabalho supracitado, durante avaliação pós-EEF, que foi de $44,4 \%$ para o paciente $1,53,8 \%$ para o paciente 2 e $32,2 \%$ para o paciente 3 .

É postulado que a maximização da produção de inputs sensoriais mediados pela estimulação do fuso neuromuscular e conseqüente movimentação dos seguimentos do membro facilitada pela EEF, associada a repetidos movimentos voluntários, induz ao mecanismo de potenciação em longo prazo em áreas corticais específicas, modificando a excitabilidade de neurônios sensoriomotores específicos e, levando assim, ao aprendizado motor19.

Quando comparados todos os resultados entre si, foi observada uma relação direta somente entre os ganhos na ADM e a melhora na escala de Fugl-Meyer. Dessa forma, fica evidente a importância da liberdade de movimento articular para realização de atividades funcionais, principalmente os que requerem grandes amplitudes de movimento articular, tais como extensão total de cotovelo para maioria das atividades de alcance e flexão superior a $90^{\circ}$ do mesmo para conduzir objetos à boca.

Alguns pontos críticos devem ser observados neste estudo. As escalas de Kendal e FuglMeyer, embora largamente utilizadas, são instrumentos subjetivos de avaliação, o que pode dar margem a interpretações diferentes entre avaliadores. A utilização de somente um avaliador independente torna a obtenção final dos resultados menos precisa e, finalmente, o baixo número da amostra não permite que sejam feitas afirmações conclusivas acerca desta nova modalidade terapêutica.

Contudo, a escala de avaliação de FuglMeyer é considerada pela maioria dos terapeutas como sendo um dos melhores instrumentos para avaliação quantitativa da funcionalidade do membro superior de pacientes vítimas de AVE, devido a alta especificidade dos sub-itens presentes no domínio para membro superior e a grande facilidade para aplicação ${ }^{20}$. Portanto, é esperado que os ganhos descritos no presente estudo sejam relevantes para melhora no desempenho das atividades da vida diária dos pacientes.

\section{CONCLUSÃO}

A aplicação de EEF com estimulação simultânea de extensores de punho e cotovelo 
mostrou-se efetiva para melhora da força mus cular, ADM passiva e funcionalidade do membro superior de pacientes hemiparéticos crônicos, entretanto, sem significativa vantagem em relação aos trabalhos prévios que utilizam estimulação somente em extensores de punho.

\section{REFERÊNCIAS BIBLIOGRÁFICAS}

1. Whisnant JP, Basford JP, Bernstein EF, Cooper ES, Dyken ML, Easton JD, et al. Special report from the rational institute of neurological disorders and stroke - Classification of cerebrovascular disease III. Stroke 1990;21:637-76.

2. Lessa I. Epidemiologia das doenças cerebrovasculares no Brasil. Rev Soc Cardiol Estado de São Paulo 1999;9:509-18.

3. Chen JC, Linag C-C, Shaw F-Z. Facilitation of sensory and motor recovery by thermal intervention for the hemiplegic upper limb in acute stroke patients. Stroke 2005;36:2665-9.

4. Colebatch JG, Gandevia SC. The distribution of muscular weakness in upper motor neuron lesion affecting the arm. Brain 1989;112:749-63.

5. Muellbacher W, Richards C, Ziemann U, Wittenberg G, Weltz D, Boroojerdi $\mathrm{B}$, et al. Improving hand function in chronic stroke. Arch Neurol 2002;59:1278-82.

6. Wu CW, Seo HJ, Cohen LG. Influence of electric somatosensory stimulation on paretic-hand function in chronic stroke. Arch Phys Med Rehabil 2006;87:351-7.

7. Gabr U, Levine P, Page S. Home-based electromyography-trigged stimulation in chronic stroke. Clin Rehabil 2005;19:737-45.

8. Chae J, Fang Z-P, Walker M, Purmehdi S. Intramuscular electromyographycally controlled neuromuscular electrical stimulation for upper limb recovery in chronic hemiplegia. Am J Phys Med Rehabil 2001;80:935-41.
9. Glanz M, Klawansky S, Stason W, Berkey C, Chalmers TC. Functional electrostimulation in poststroke rehabilitation: A meta-analysis of the randomized controlled trials. Arch Phys Med Rehabil 1996;77:54953.

10. Kraft GH, Fitts SS, Hammond MC. Techniques to improve function of arm and hand in chronic hemiplegia. Arch Phys Med Rehabil 1992;73:220-7.

11. Braker LL, Yeh C, Wilson D, Waters RL. Electrical stimulation of wrist and fingers for hemiplegic patients. Phys Ther 1979;59:1495-9.

12. Hummelsheim $\mathrm{H}$, Maier-Loth $\mathrm{ML}$, Eickhof $C$. The functional value of electrical muscle stimulation for the rehabilitation of the arm in stroke patients. Scand J Rehabil Med 1997;29:3-10.

13. Nudo R. Adaptive plasticity in motor córtex: implications for rehabilitation after brain injury. J Rehabil Med 2003;41:7-10.

14. Kots YM, Syrovegin AV. Fixed set of variants of interactions of the muscles to two joints in the execution of simple voluntary movements In: Shumway-Cook A, Woollacott MH. Controle Motor - teoria e aplicações práticas. 2a ed., São Paulo: Manole, 2003, 443p.

15. Palmer ML, Epler ME. Técnicas de avaliação músculo-esquelética. 2.ed., Rio de Janeiro: Guanabara, 1998, 21p.

16.Shumway-Cook A, Woollacott MH. Controle motor: Teoria e aplicações práticas. 2a ed., São Paulo: Manole, 2003, 127p.

17. Carr J, Shepherd R. Ciência do Movimento. 2a ed., São Paulo: Manole, 2003, 56-64.

18. Kroon JR, ljzerman MJ, Chae J, Lankhrst GJ, Zilvold G. Relation between stimulation characteristics and clinical outcomes in studies using electrical stimulation to improve motor of the upper limb extremity in stroke. J Rehabil Med 2005;37:65-74.

19. Powell J, Pandyan D, Granat M, Cameron M, Stott D. Electrical stimulation of wrist in poststroke. Stroke 1999;30:1384-9.

20. Gladstone DJ, Danells CJ, Black SE. The fugl-meyer assessment of motor recovery after stroke: A critical review of its measurement proprieties. Neurorehabil Neural Repair 2002;16:232-40. 